\title{
Measurement of the surface potential decay of corona-charged polymer films using the pulsed electroacoustic method
}

\author{
G Chen ${ }^{1}, \mathrm{Z} \mathrm{Xu}^{1}$ and $\mathrm{L} \mathrm{W} \mathrm{Zhang}^{2}$ \\ ${ }^{1}$ School of Electronics and Computer Science, University of Southampton, UK \\ ${ }^{2}$ Institute of Fluid Physics, CAEP PO Box 919-150, Mianyang 621900, \\ People's Republic of China
}

Received 12 December 2006, in final form 31 January 2007

Published 20 March 2007

Online at stacks.iop.org/MST/18/1453

\begin{abstract}
In this paper, the pulsed electroacoustic (PEA) technique that allows the determination of space charge in a dielectric material has been used to monitor the electrical potential decay of corona-charged polyethylene films of different thicknesses. To prevent possible disturbance of the surface charge during the PEA measurements, two thin polyethylene films were placed on both sides of the corona-charged sample. Charge profiles measured at different times were used to calculate the potential across the sample. The obtained potential decay was compared with the potential measured using the conventional method. Good agreement has been obtained. More importantly, the charge profile obtained using the PEA technique indicates that bipolar charge injection has taken place.
\end{abstract}

Keywords: corona charging, surface potential decay, polyethylene, pulsed electroacoustic technique, bipolar charge injection

(Some figures in this article are in colour only in the electronic version)

\section{Introduction}

Over the years, considerable interest has been shown in the surface potential decay of corona-charged polymeric materials. The corona charging technique has widespread application in the preparation of polymer foils for the use in electret microphones [1]. On the other hand, measurement of the potential decay has been proven to be a simple and useful technique for characterizing insulating materials and the charging method. For example, this method enables a convenient determination of charge carrier mobility and trap parameters. One of the well-known effects in the observation of surface potential decay is the crossover phenomenon [2], i.e. initially a sample charged to a high-potential decays more rapidly than one charged to a lower potential. Ignoring this phenomenon, most authors report that charge decay plots show the same general features: an initial rapid surface potential decay followed by a much slower decay. However, the physics of charge decay is not adequately understood based on the existing experimental results. Various mechanisms have been proposed using different assumptions. Generally, there are three possible routes for charge decay in corona-charged polymer, i.e. through the atmosphere, along the surface and transport through the bulk. The former two routes may not be important as the surface potential decay is highly dependent on the thickness [3]. The surface conduction may only become important when high humility is encountered. Consequently, the majority of theories and models [4-7] for surface potential decay have been developed in terms of bulk transport. In addition, a polarization process has also been proposed to explain the potential decay in the case of polar polymeric materials.

Over the last 20 years, there has been significant development in space charge mapping in solid dielectrics due to advances in sensors, signal capture and processing. Techniques such as the laser-induced pressure pulse method (LIPP) [8], thermal step method (TS) [9] and pulsed electroacoustic method (PEA) [10] have been utilized to observe charge dynamics in dielectric materials. Significant progress has been made in understanding charge generation, 
transport, trapping and conduction. The aim of the present paper is to utilize the space charge measurement technique to observe the charge decay process in a corona-charged polymeric material. The anticipation is that the application of this charge mapping technique may shed more light on the mechanism of charge decay.

In the present study, low-density polyethylene samples charged with a corona method to very high negative surface potentials are investigated using the PEA technique. We first describe the sample preparation and experimental setup. Then, the results of surface potential decay obtained using two different methods from two samples with different arrangements are presented and discussed. Finally, conclusions with respect to surface charge decay are drawn.

\section{Experimental details}

\subsection{Sample preparation}

Low-density polyethylene (LDPE) thin films with different thicknesses were purchased commercially from GoodFellow. Additive-free LDPE was selected to avoid extra complications that may be caused by the presence of antioxidants, etc. The film was cut into a circular disc with a diameter of $50 \mathrm{~mm}$, cleaned initially using methanol, rinsed in deionized water and then dried by air. Care has to be taken when two layers are involved. We started from one side of the edge and attached films together towards the other side. In the presence of air bubbles, light pressure was used to remove them. PEA measurement revealed that there was no extra peak appearing in the middle of the two-layered sample, indicating good contact between the films. This also suggests that the generation of tribocharge in the present case seems not to be a major issue compared with the amount of charge formed due to corona charging.

\subsection{Corona setup and potential measurement}

The LDPE film was negatively charged in a corona setup consisting of a high voltage needle electrode, a wire mesh grid and an earthed electrode. The needle voltages of $-17 /-11 \mathrm{kV}$ and mesh voltages of $-8 /-4 \mathrm{kV}$ were used respectively. The surface potential is determined by the mesh voltage. However, the reduction in the needle voltage is necessary. If the voltage on the needle were kept at $-17 \mathrm{kV}$, the voltage on the mesh would exceed $-4 \mathrm{kV}$ due to its relative position in the electrode system. The charging time is typically around $2 \mathrm{~min}$. A schematic diagram of the corona discharge setup is shown in figure 1. Both temperature and relative humidity will influence the electric charge decay. To achieve consistent results, all experiments were carried out in a controlled environment where temperature and relative humidity were $21^{\circ} \mathrm{C}$ and $45 \%$ respectively.

After that, the sample was transferred quickly to the compact JCI 140 static monitor to observe the isothermal surface potential decay. The time required to transfer the sample for potential measurement after corona charging is around $1 \mathrm{~s}$. The JCI 140 is a compact electric field mill instrument that allows one to determine the voltage of a surface at a distance [11]. It is an electromechanical device which measures the strength of a static electric field. One or more

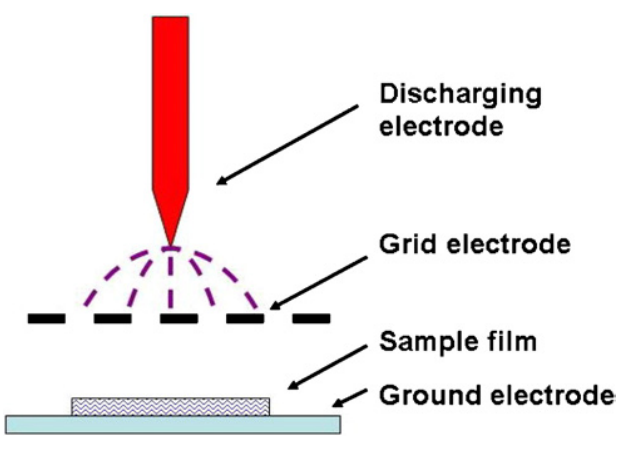

Figure 1. Corona discharge setup.

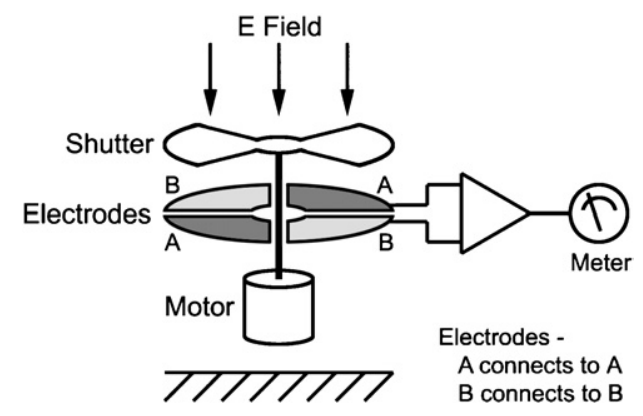

Figure 2. Schematic diagram of the field mill.

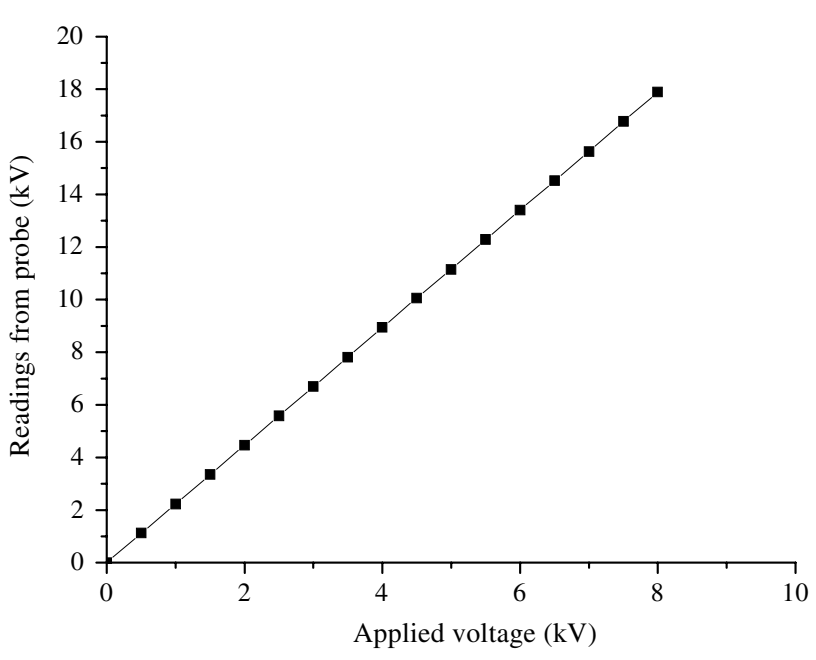

Figure 3. Calibration for the JCI 140 probe.

electrodes are alternately exposed to and then shielded from the field to be measured. The electric current which flows to and from the electrodes is proportional to the strength of the electric field. Figure 2 shows the schematic diagram of the field mill.

The readings from the JCI 140 static monitor are not direct values of the surface potential, but proportional to the surface potential. To convert the readings into the surface potential, a calibration has to be carried out. In the present case, a thin aluminium foil attached to the surface of a sample was connected to a dc voltage supply. Readings were taken when varying voltages were applied. A linear relationship was obtained between the readings and the applied voltage as shown in figure 3 . 


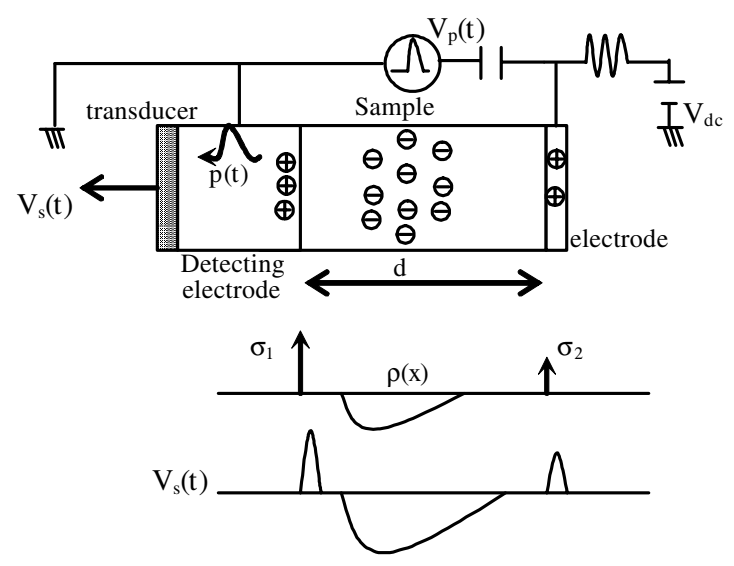

Figure 4. Schematic diagram of the PEA technique.

\subsection{PEA method}

The PEA technique is more common than the other two techniques due to its simple structure, low cost and ease of implementation. A schematic diagram of a conventional PEA system is shown in figure 4. A high voltage pulse of $5 \mathrm{~ns}$ length is applied to the sample sandwiched between the two electrodes. The pulse electric field produced interacts with charge layers, generating an electric force which displays charge. The consequence is the formation of pulsed acoustic waves corresponding to each charge layer with respect to neutrality. The resultant acoustic signals are detected by a piezo-electric transducer, so that the charge distribution in the sample under test can be obtained from the output voltage profile of the transducer. The electric signal obtained in the time domain represents the charge distribution [10]:

$$
V_{s}(t)=K\left[\sigma_{1}(0)+\sigma_{2}\left(d / v_{\mathrm{sa}}\right)+v_{\mathrm{sa}} \Delta T \rho\left(x=v_{\mathrm{sa}} t\right)\right] e_{p},
$$

where $\sigma_{1}$ and $\sigma_{2}$ are the surface charges at the electrodes, $v_{\mathrm{sa}}$ is the sound velocity through the material, $d$ is the sample thickness, $\Delta T$ is the width of the pulse, $\rho$ is the bulk charge and $e_{p}$ is the amplitude of the pulse voltage. The analysis of space charge profiles is restricted to one dimension.
A thin semiconducting film (LDPE loaded with carbon black) was attached to the top copper electrode to improve acoustic matching. A very thin layer of silicone oil was applied to the interface between the electrodes and the sample to achieve better acoustic transmission. The quantitative charge analysis needs $K$ to be calibrated and this is typically done by applying a small voltage across the sample to generate a known charge density on the two surfaces at the electrodes. The procedure is termed calibration and more details can be found in our published work [12].

Once the charge density distribution $\rho(x)$ in the sample is known, it is possible to calculate the electric field across the sample based on Poisson's equation:

$$
\frac{\mathrm{d} E(x, t)}{\mathrm{d} x}=\frac{\rho(x, t)}{\varepsilon_{0} \varepsilon_{r}}
$$

where $\varepsilon_{0}$ is the permittivity of vacuum and $\varepsilon_{r}$ is the relative permittivity of LDPE.

The potential across the sample can then be estimated by

$$
V(t)=-\int_{0}^{d} E(x, t) \mathrm{d} x,
$$

where $d$ is the thickness of the sample. However, in the present case, the potential was calculated including the charge contribution from the first charge peak, imaging charge on the bottom electrode, in order to compare with the potential measured using the conventional method.

\section{Results and discussion}

After charges were deposited via the corona charging process, the surface potential was monitored. The potential decay of the corona-charged LDPE film with a thickness of $180 \mu \mathrm{m}$ is shown in figure 5. Here, the absolute surface potential is plotted. It can be seen that the surface potential decreases with time and the decay rate is very slow. The decay has been attributed to various mechanisms including recombination with opposite ions in air, surface migration and bulk conduction [2]. The bulk process has been widely accepted and several models have been proposed to explain the surface potential decay [4-7].

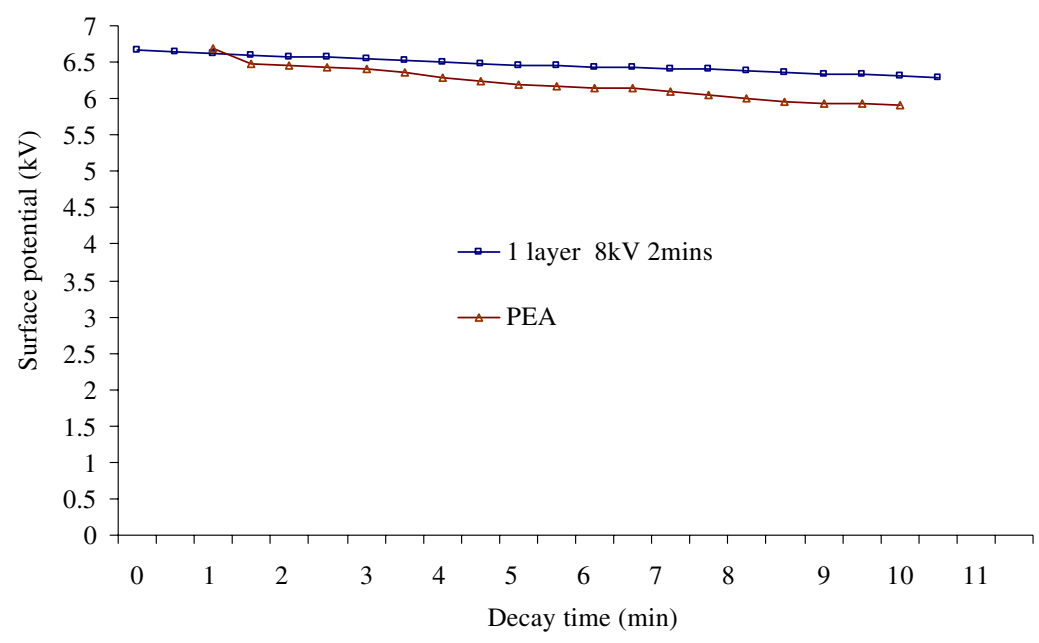

Figure 5. Surface potential decay after corona-charged LDPE $(-8 \mathrm{kV}, 180 \mu \mathrm{m})$. 


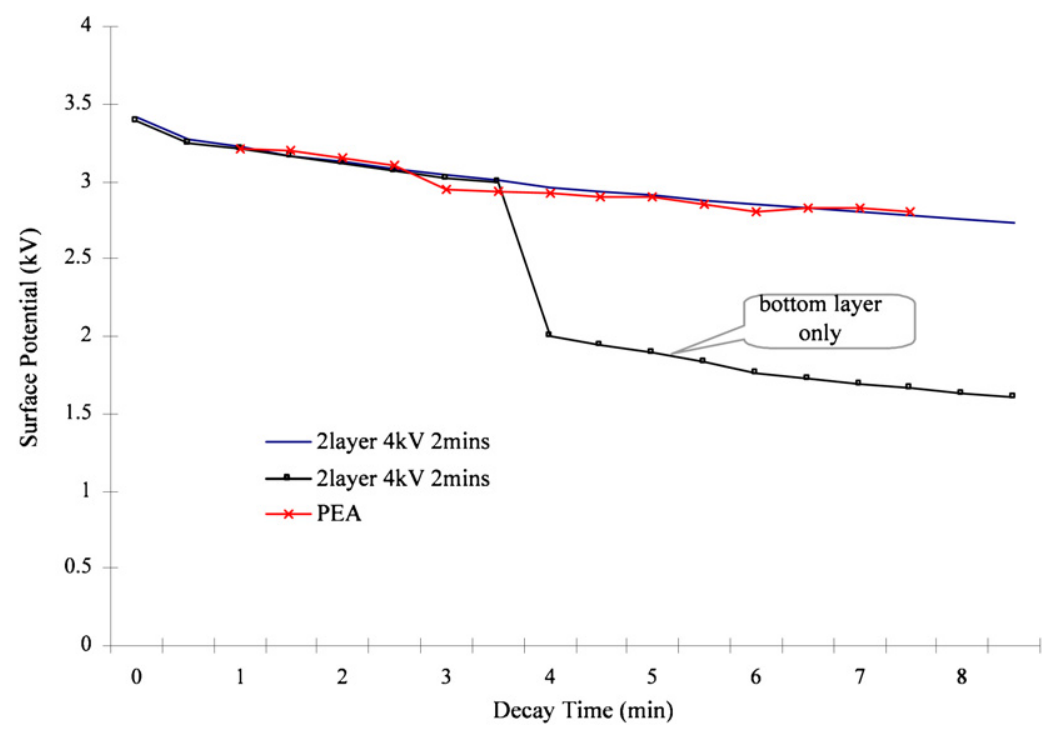

Figure 6. Potential decay of a two-layer sample $(-4 \mathrm{kV}, 50 \mu \mathrm{m}+50 \mu \mathrm{m})$.

Figure 6 shows the surface potential decay of two-layer $50 \mu \mathrm{m}$ LDPE films. To achieve a similar electric field, the grid potential was set to $-4 \mathrm{kV}$ in this case. Again, the surface potential shows a slow decay. In another test, after the surface potential was monitored for $4 \mathrm{~min}$, the top layer was carefully removed and the surface potential produced by the bottom layer was continuously observed as shown in the figure. The surface potential could still be observed and it shows a similar decay trend. The reading from the JCI 140 is affected by the distance to the sample. One layer of the LDPE film is only $50 \mu \mathrm{m}$ thick and this is negligible compared to the $18 \mathrm{~mm}$ separation distance between the probe and the ground electrode. So the removal of the top layer has little effect on the reading in the present study. Since the surface potential is a representation of both surface charge and bulk charge, this implies that there is electric charge present either on the top surface of the bottom layer or in the bulk. In either case, charge has to come from the charge injection. The removal of the top film may have two effects. Firstly, it may lead to charge transfer between the two layers. Secondly, it may modify the charge distribution in the presence of charge. Due to the two layers consisting of the same LDPE, charge transfer during separation is not significant. This is confirmed by the surface potential measurement of the two-layered fresh sample (without corona charge). The surface potential obtained after the removal of the top layer was less than $0.01 \mathrm{kV}$, therefore making little contribution to the observed surface potential. The influence on the existing charge distribution of the removal of the top layer is difficult to assess. However, the trend reported in figure 6 has been consistently observed.

It is clear to us that bulk charge transport is responsible for the surface potential decay. As the surface potential changes relatively slowly, it is possible to monitor charge evolution using the space charge measurement.

Figure 7 shows the space charge distribution in a negatively corona-charged $180 \mu \mathrm{m}$ LDPE film. The time to transfer the corona-charged sample to the PEA system takes 1 to $2 \mathrm{~min}$ as the sample has to be manually introduced into the system. To minimize the disturbance to the deposited charge, both surfaces of the sample were protected by an extra layer $50 \mu \mathrm{m}$ LDPE film. There are four major peaks. The first and fourth peaks correspond to the induced charges on the PEA electrodes while the second and third peaks are charges formed in the sample during corona charging. Polarization is unlikely to be responsible for the observed peaks as the material used is nonpolar. Negative charge is clearly present at the top surface. This is expected as a process of charge deposition. Surprisingly, a layer of positive charge is observed at the bottom surface of the sample. This implies that double injection has taken place [13]. Charge injection has been observed to occur in LDPE above a threshold value of $10 \mathrm{kV} \mathrm{mm}^{-1}$ [14]. From the surface potential in figure 5, the electric field experienced by the sample is in the range of $40 \mathrm{kV} \mathrm{mm}-1$, well above the threshold value for charge injection. So the observed bipolar charge injection in coronacharged LDPE is real. The charge profiles over a period of time are shown with different colours. It can be seen that charge decreases with time as indicated by arrows.

$V(t)$ obtained from space charge measurements is shown in figure 3. It is very close to the potential monitored by the JCI 140 static monitor. This implies that the potential measured using the PEA method is reliable. There is a slight difference in potential obtained by the two methods. There are two possible reasons. As mentioned in our previous section, to obtain quantitative charge information, a calibration has to be carried out when using the PEA technique. Ideally, the calibration should be performed on the same sample to avoid an error introduced by assembling the sample into the system [12]. This is impractical for the corona-charged sample. As a result, a similar sample arrangement was used to calibrate the corona-charged sample. This may lead to a small error. On the other hand, the insertion of two $50 \mu \mathrm{m}$ LDPE films attached to the corona-charged film may also be a source of error.

Figure 8 shows the space charge distribution and dynamics in the two-layer LDPE sample. Again, to protect the deposited charge, two $50 \mu \mathrm{m}$ LDPE films were attached to both the surfaces separately. Compared with the space charge 


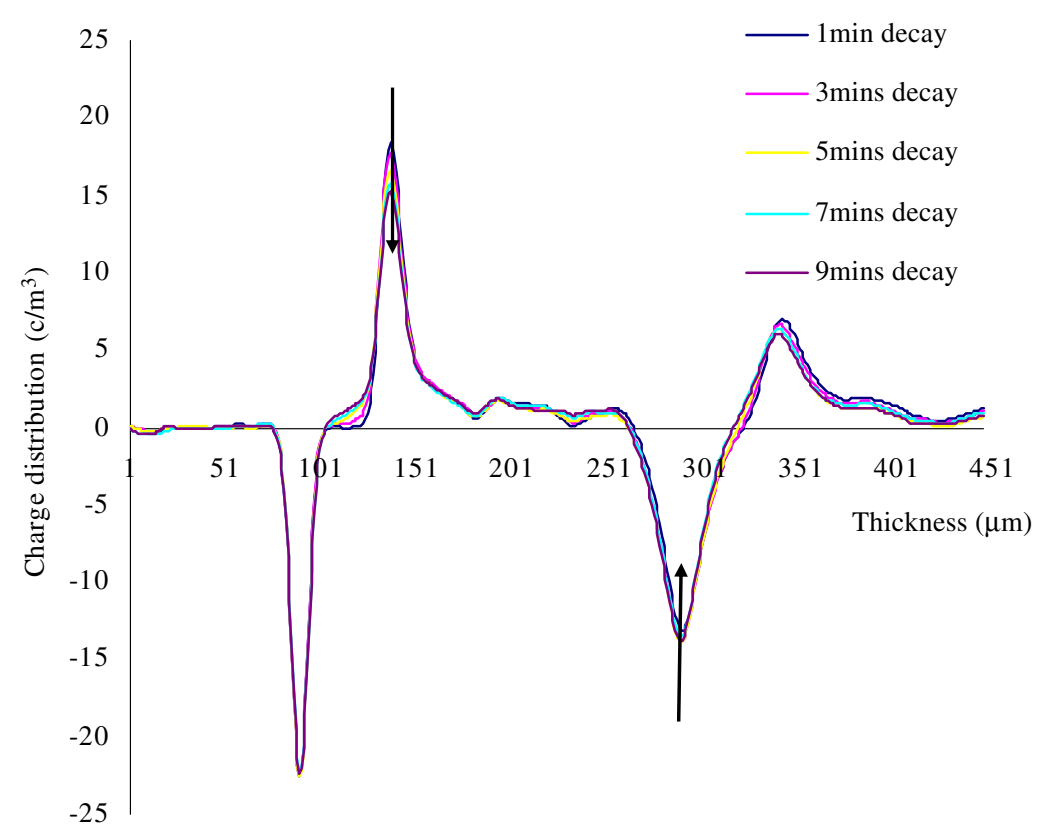

Figure 7. Space charge distribution and evolution in corona-charged $180 \mu \mathrm{m}$ thick LDPE.

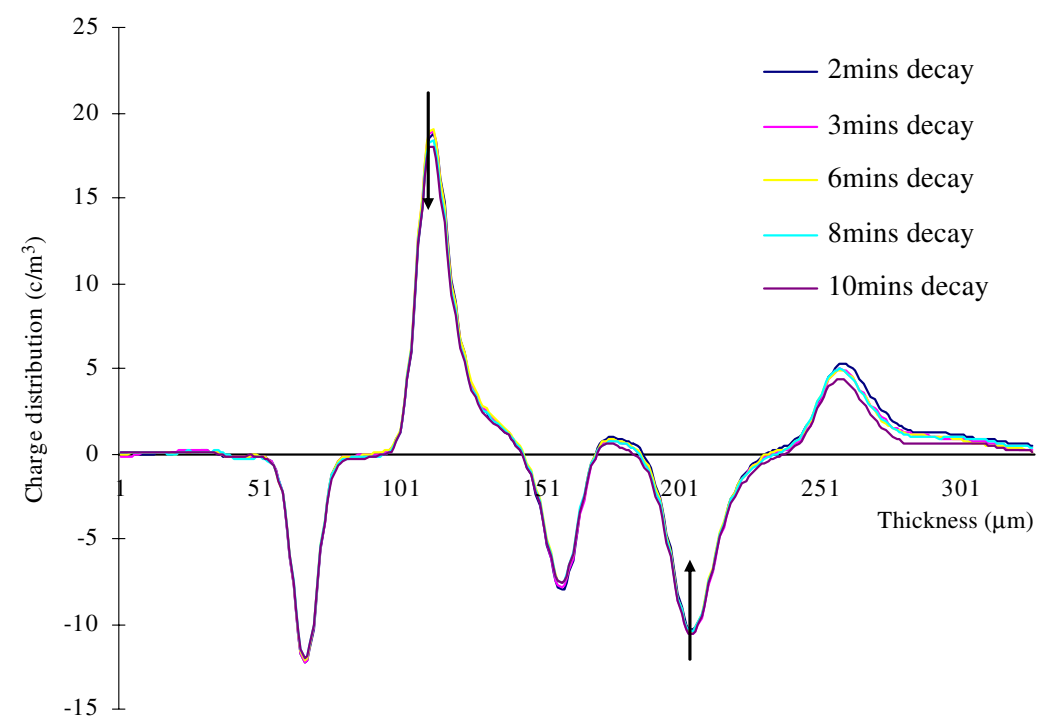

Figure 8. Space charge distribution and evolution in two-layer $(50 \mu \mathrm{m}+50 \mu \mathrm{m}) \mathrm{LDPE}$.

distribution in the $180 \mu \mathrm{m}$ LDPE film, an extra negative charge peak is observed at the interface between the two layers. This negative charge is believed to be formed due to charge injection from surfaces and then transported to the interface. The appearance of negative charge at the interface is consistent with the early potential result obtained from the bottom layer of the two-layer LDPE sample as shown in figure 6. The charge injection and bulk transport processes are responsible for the surface potential decay in corona-charged LDPE. Following the same procedure as described in the previous section, the surface potential can be calculated based on space charge measurements. $V(t)$ in two-layer LDPE is shown in figure 6 . The surface potentials obtained by the two methods almost overlap.
Generally, the surface potential is formed during corona charging with a specific voltage polarity; therefore, all the exiting models are established based on a single type of charge carrier [4-7]. By using the PEA technique, we are able to reveal that bipolar charge carrier injection has taken place. These new results challenge the existing surface potential decay models. Further research on corona-charged polymeric materials using the space charge mapping technique may allow one to investigate charge transport processes and establish a new model taking into consideration bipolar charge injection. It is likely that recombination between bipolar charge carriers may be responsible for the surface potential decay. Our recent work on the charge decay in LDPE after the removal of high dc fields [13] indicates that fast charge decay occurs when a 
higher dc field is used. This phenomenon may have the same origin as the crossover effect observed.

\section{Conclusion}

The surface potential decay of corona-charged LDPE films has been studied using both the conventional surface potential method and the PEA technique. Compared with the conventional surface potential measurement, the PEA technique provides a good, consistent potential across the sample. More importantly, the PEA technique is capable of showing a clear charge profile in corona-charged LDPE. The charge profiles obtained strongly suggest that bipolar charge carrier injection has occurred. The new surface potential decay model is required to describe charge transport processes in corona-charged LDPE. It is believed that recombination between two types of charge carriers injected may be responsible for the surface charge decay.

\section{References}

[1] Sessler G M 1987 Electrets Topics in Applied Physics vol 33 2nd edn (Berlin: Springer)

[2] Ieda M, Sawa G and Shinohara U 1967 A decay process of surface electric charges across polyethylene film Japan. $J$. Appl. Phys. 6 793-4

[3] Zhang L W, Xu Z and Chen G 2007 Charge decay of corona-charged low density polyethylene Electrostatics 2007 (Oxford, UK)
[4] Sonnonstine T J and Perlman M M 1975 Surface-potential decay in insulators with field-dependent mobility and injection efficiency J. Appl. Phys. 46 3975-81

[5] Wintle H J 1972 Surface-charge decay in insulators with non-constant mobility and with deep trapping J. Appl. Phys. $432927-30$

[6] Baum E A, Lewis T J and Toomer R 1977 Decay of electrical charge on polyethylene films J. Phys. D: Appl. Phys. 10 487-97

[7] Berlepsch H von 1985 Interpretation of surface potential kinetics in HDPE by a trapping model J. Phys. D: Appl. Phys. 18 1155-70

[8] Lewiner J 1986 Evolution of experimental techniques for the study of the electrical properties of insulating materials IEEE Trans. Electr. Insul. 21 351-60

[9] Cherifi A, Abou-Dahka M and Toureille A 1992 The validation of the thermal step method IEEE Trans. Dielectr. Electr. Insul. 27 1152-8

[10] Li Y, Yasuda M and Takada T 1994 Pulsed electroacoustic method for measurement of charge accumulation in solid dielectrics IEEE Trans. Dielectr. Electr. Insul. 1 188-95

[11] Chubb J N 1990 Two new designs of 'field mill' type fieldmeters not requiring earthing of rotating choppers IEEE Trans. Ind. Appl. 26 1178-81

[12] Chen G, Chong Y L and Fu M 2006 Calibration of the pulsed electroacoustic technique in the presence of trapped charge Meas. Sci. Technol. 17 1974-80

[13] Chen G and Xu Z 2007 Space charge dynamics in low density polyethylene under high dc electric fields Electrostatics 2007 (Oxford, UK)

[14] Montanari G C, Mazzanti G, Palmieri F, Moton A, Perego G and Serra S 2001 Space-charge trapping and conduction in LDPE, HDPE and XLPE J. Phys. D: Appl. Phys. 34 2902-11 